\title{
A Possible Mechanism Underlying an Antidepressive-Like Effect of Kososan, a Kampo Medicine, via the Hypothalamic Orexinergic System in the Stress-Induced Depression-Like Model Mice
}

\author{
Naoki Ito, ${ }^{a}$ Takeshi Yabe, ${ }^{a, b}$ Takayuki Nagai, ${ }^{a, b}$ Tetsuro Oikawa, ${ }^{a}$ Haruki YAmadA, ${ }^{a, b}$ and \\ Toshihiko HANAWA $*, a$ \\ ${ }^{a}$ Department of Clinical Research, Oriental Medicine Research Center, Kitasato University; Tokyo 108-8642, Japan: and \\ ${ }^{b}$ Kitasato Institute for Life Sciences \& Graduate School of Infection Control Sciences, Kitasato University; Tokyo \\ 108-8641, Japan. Received June 3, 2009; accepted July 13, 2009; published online July 14, 2009
}

Kososan, a Kampo (Japanese herbal) medicine, has an antidepressive-like effect in behavioral animal models of depression and has been used clinically for the improvement of depressive mood. However, mechanism(s) underlying the antidepressive-like effect of kososan remain unknown. Previous studies showed that orexin-A (OX-A), a neuropeptide that is involved in feeding and arousal, exhibits an antidepressive-like property via hippocampal cell proliferation. Here, we used immunohistochemical analysis with bromodeoxyuridine (BrdU), a marker of proliferating cells, to investigate the effect of long-term treatment with kososan on the orexinergic system and on hippocampal cell proliferation. Oral administration of kososan $(1.0 \mathrm{~g} / \mathrm{kg})$ or milnacipran $(60 \mathrm{mg} / \mathrm{kg})$, a serotonin and noradrenaline reuptake inhibitor, for $28 \mathrm{~d}$ led to an antidepressive-like effect in the stress-induced depression-like model mice and reversed the stress-induced decrease in the number of OX-A-positive cells in the lateral hypothalamic area. In addition, both kososan and milnacipran alleviated the stress-induced decrease in the number of BrdU-positive cells in the hippocampal dentate gyrus. Moreover, the antidepressive-like effect and the increase in cell proliferation and in the number of neuropeptide Y (NPY, which is closely associated with orexinergic system)-positive cells in the dentate gyrus induced by kososan were blocked by treatment with SB-334867, an orexin receptor 1 antagonist. These results suggest that kososan exerts an antidepressive-like effect via the improvement of the stress-induced decrease in hippocampal cell proliferation and that the mechanism underlying the antidepressive-like effect of kososan, but not of milnacipran, may be associated with the regulation of orexinergic and/or NPYergic transmission.

Key words kososan; Kampo; antidepressive-like effect; orexin-A; cell proliferation; neuropeptide Y

Kososan, a Kampo (Japanese herbal) medicine (Xiang-SuSan in Chinese), is composed of five herbs (Cyperi Rhizoma, Perillae Herba, Aurantii Nobilis Pericarpium, Glycyrrhizae Radix, and Zingiberis Rhizoma) and is clinically used to treat the depression-like symptoms associated with the initial stage of the common cold, anorexia, food-related allergic urticaria, irritable bowel syndrome, chronic fatigue syndrome, insomnia, and autonomic imbalance. It has been also clinically suggested that kososan can alleviate the depression induced by interferon (IFN)- $\alpha$ therapy for hepatitis C. ${ }^{1)}$ Our previous studies using two animal models of depression, stress- and IFN- $\alpha$-induced depression-like model mice, demonstrated that oral administration of kososan leads to an antidepressive-like effect via the normalization of the dysfunction of the hypothalamic-pituitary-adrenal (HPA) axis, which is strongly associated with the pathogenesis of depression. ${ }^{2,3)}$ These findings provide supporting evidence that kososan may be beneficial in the treatment of depression.

Depressive disorder is thought to reflect maladaptive changes in stress-responsive systems. ${ }^{4)}$ Numerous studies have reported that excessive exposure to stressful life events induces the onset of depression, which is accompanied by a decrease in neurogenesis in the dentate gyrus of the hippocampus. $^{5-8)}$ Therefore, disruption of neurogenesis plays a crucial role in the mechanism by which stress facilitates depression. ${ }^{9,10)}$ Furthermore, chronic treatment with antidepressants increases neurogenesis in the rat hippocampus ${ }^{11)}$ and adult hippocampal neurogenesis is required for the behavioral recovery effects of antidepressants. ${ }^{12)}$ Thus, neurogenesis-inducing compounds may directly and/or indirectly pro- duce antidepressive-like effects.

Recent studies have reported that orexin-A (OX-A), which is a neuropeptide produced especially in the lateral hypothalamic area (LHA) and in the posterior hypothalamus, ${ }^{13,14)}$ is involved in the pathology of depression. A decrease in the number and size of OX-A-producing neurons in the LHA was observed in male Wistar-Kyoto rats, which exhibit a hyper-responsiveness of the HPA axis, a characteristic of depression. ${ }^{15}$ Suicidal patients with major depression exhibit significantly lower OX-A levels in the cerebrospinal fluid when compared with patients who suffer from adjustment disorder and dysthymia. ${ }^{16)}$ In addition, our previous study demonstrated that intracerebroventricular administration of OX-A induces an antidepressive-like effect via hippocampal cell proliferation in mice. ${ }^{17)}$ The orexin receptor 1 (OXR1), which is a subtype of the orexin receptor, is expressed in the granule cell layer, hilus, and CA1-3 regions of the hippocampus. ${ }^{18)}$ In addition, orexin-producing neurons also project to the hippocampus, in which neurogenesis occurs. Therefore, hippocampal neurogenesis may, at least in part, be regulated by a change in OX-A expression in the LHA. The neuropeptide Y (NPY) is implicated in the stimulation of feeding behavior ${ }^{19)}$ and its effect is similar to that of OX-A. NPY-producing neurons are distributed not only in the arcuate nucleus, but also in the hippocampus (in interneurons of the hilus and in CA1-3 regions). ${ }^{20)}$ Several studies have also shown that the levels of NPY in the cerebrospinal fluid are decreased in patients with major depression, ${ }^{21-23)}$ that NPY exhibits an antidepressive-like activity via the Y1 receptor in vivo, ${ }^{24)}$ and that NPY exerts a proliferative activity in neural 
precursor cells in vitro. ${ }^{25)}$ Several lines of evidence among these findings suggest that dysfunction of the orexinergic and NPYergic systems may be associated with the pathology of depression and decrease in neurogenesis.

Although several traditional herbal medicines are effective for the treatment of depressive mood, their detailed mechanism of action is not fully understood. Recently, the total flavonoids extracted from Xiaobuxin-Tang, which is a traditional Chinese herbal medicine composed of four herbs such as Haematitum, Flos Inulae, Folium Phyllostachydis Henonis and Semen Sojae Preparatum, were shown to exert an antidepressive-like activity in animal models of depression, such as the forced swimming test and learned helplessness, ${ }^{26)}$ and to upregulate the stress-induced decrease in hippocampal neurogenesis in chronically stressed rats. ${ }^{27)}$ However, it has not been investigated whether the antidepressive-like effect of kososan is involved in the regulation of hippocampal cell proliferation and of the hypothalamic orexinergic and hippocampal NPYergic systems.

In the present study, we aimed to assess the role of the orexinergic and/or NPYergic systems in the antidepressivelike effect of kososan by investigating the regulatory effects of long-term (28 d) treatment with kososan on these systems and on hippocampal cell proliferation, which corresponds to the developmental stage of neurogenesis, in the stressinduced depression-like model mice. The results of these studies lead us to propose a hypothesis to explain the differences in the mechanisms underlying the antidepressive-like effects of kososan and of the antidepressant milnacipran.

\section{MATERIALS AND METHODS}

Animals We used seven-week-old male ddY mice (Japan SLC, Hamamatsu, Japan) that weighed 35-40 g at the beginning of the experiment. The mice were housed under conditions of constant temperature $\left(23 \pm 2^{\circ} \mathrm{C}\right)$ and humidity $(55 \pm 10 \%)$, with food and water available ad libitum (unless otherwise specified) and with a 12/12 h light-dark cycle $(08 \mathrm{~h} 00$ to $20 \mathrm{~h} \mathrm{00)}$ during the stress procedure. All animal experiments were performed according to the Guidelines for Care and Use of Laboratory Animals of Kitasato University.

Drugs The component herbs of kososan were as follows: Cyperi Rhizoma (rhizome of Cyperus rotundus L.), $4.0 \mathrm{~g}$ (Lot No. AE7951, Tsumura \& Co., Tokyo, Japan); Perillae Herba (leaf of Perilla frutescens BRITTON var. acuta KUDO), $2.0 \mathrm{~g}$ (Lot No. B04401, Tsumura \& Co.); Aurantii Nobilis Pericarpium (pericarp of Citrus unshiu MARKovich), $3.0 \mathrm{~g}$ (Lot No. AD7971, Tsumura \& Co.); Glycyrrhizae Radix (root of Glycyrrhiza uralensis FISHER), $2.0 \mathrm{~g}$ (Lot No. 8661621, Uchida Wakan-yaku Co., Ltd., Tokyo, Japan), and Zingiberis Rhizoma (rhizome of Zingiber officinale RosCOE), $0.5 \mathrm{~g}$ (Lot No. AK8761, Tsumura \& Co.). Specimens were deposited at Oriental Medicine Research Center, Kitasato University, Japan. The Kampo formula was decocted with $600 \mathrm{ml}$ of distilled water until the volume was reduced to half and the extract was filtrated through filter paper immediately in vacuo. The filtrate was lyophilized and the yield of kososan extract was approximately $28 \%$ of the herbal mixture, based on its dry weight. This extract was used in the present study. Toledomin ${ }^{\mathbb{B}}$ (milnacipran hydrochloride) was purchased from Asahi Kasei Pharma Corporation (Tokyo, Japan).

Drug Treatments The kososan extract or milnacipran was dissolved or suspended in distilled water, respectively. Our previous studies demonstrated that oral administration of kososan or milnacipran leads to an antidepressive-like effect in the stress-induced depression-like model mice when administered at the dose of $1.0 \mathrm{~g} / \mathrm{kg}$ or $60 \mathrm{mg} / \mathrm{kg}$, respectively. ${ }^{2}$ Therefore, kososan $(1.0 \mathrm{~g} / \mathrm{kg})$ or milnacipran $(60 \mathrm{mg} / \mathrm{kg}$, used here as a positive control) were administered orally once daily for $28 \mathrm{~d}$.

Bromodeoxyuridine (BrdU) (Roche Diagnostics, Indianapolis, IN, U.S.A.), which is a marker of dividing cells, such as neural progenitor cells, was dissolved in saline containing $0.007 \mathrm{~N} \mathrm{NaOH}$. For experiments designed to measure cell proliferation, BrdU $(200 \mathrm{mg} / \mathrm{kg})$ was injected once at $4 \mathrm{~h}$ before brain fixation. SB-334867 (Tocris Bioscience, Ellisville, MO, U.S.A.), which is a selective OXR1 antagonist, was dissolved in saline containing $10 \%(\mathrm{w} / \mathrm{v})$ hydroxypropyl$\beta$-cyclodextrin (Tokyo Chemical Industry Co., Ltd., Tokyo, Japan) and $1 \%(\mathrm{v} / \mathrm{v})$ dimethyl sulfoxide (Sigma, St. Louis, MO, U.S.A.). SB-334867 (15 mg/kg) was injected six times once daily on days $18,25,32,35,37$, and 39 .

Preparation of Stress-Induced Depression-Like Model Mice The stress-induced depression-like model mice ${ }^{2)}$ were prepared using a combination of the modified forced swimming $(\mathrm{FS})^{28,29)}$ and the chronic mild stress ${ }^{30,31)}$ approaches (Table 1). Briefly, the mice were individually placed in separate 51 glass beakers (height, $27 \mathrm{~cm}$; diameter, $18 \mathrm{~cm}$ ) filled with 41 of water $\left(23 \pm 1^{\circ} \mathrm{C}\right)$ for $15 \mathrm{~min}$ on Day 0 . Beakers were separated by non-transparent panels to prevent the mice from seeing each other. After $15 \mathrm{~min}$ in the water, mice were removed and allowed to dry with a drier before being returned to their home cages. After $2 \mathrm{~d}$, mice were exposed to three different stress situations: tilting of the cage 30 degrees from the horizontal on Days 2 and 9, pouring of $200 \mathrm{ml}$ of water onto the sawdust bedding of the cage on Day 5 , and shaking of the cages at $200 \mathrm{rpm}$ using a Green S. Seriker II apparatus (Vision Scientific, Kyunggi, Korea) on Day 7. These stress situations were applied for 48, 24, and $24 \mathrm{~h}$, respectively, with $24 \mathrm{~h}$ intervals. Mice were then placed in 51 beakers with 41 of water for $5 \mathrm{~min}$ at $1 \mathrm{~h}$ after cage tilting; FS was performed on Day 11. Individual immobility times during the $5 \mathrm{~min}$ swim were used to assign animals to the various test groups, so that the mean immobility times were similar in all groups. This procedure was used to reduce the initial variability among groups. A mouse was considered to be immobile when it ceased struggling and remained floating motionless in the water, making only those movements

Table 1. Experimental Schedule of Stress Exposure

\begin{tabular}{rlc}
\hline \hline Day & \multicolumn{1}{c}{ Exposure of stress } & Exposure time \\
\hline 0 & Forced swimming & $15 \mathrm{~min}$ \\
2 & Cage tilting (30 degrees) & $48 \mathrm{~h}$ \\
5 & Soiled cage & $24 \mathrm{~h}$ \\
& $\quad$ (200 ml of water onto sawdust bedding) & \\
7 & Cage shaking (200 rpm) & $24 \mathrm{~h}$ \\
9 & Cage tilting (30 degress) & $48 \mathrm{~h}$ \\
11 & Forced swimming & $5 \mathrm{~min}$
\end{tabular}

Detail explanation represents in Materials and Methods. 
necessary to keep its head above water, as assessed by manual measurement using a video camera.

On Day 39, mice were again placed in 51 beakers with 41 of water at $1 \mathrm{~h}$ after the final drug treatment, and the total duration of immobility during the 5 min forced swimming test (FST) was measured. This behavioral experiment was carried out between $13 \mathrm{~h} 00$ and $16 \mathrm{~h} 00$.

Open Field Test The spontaneous locomotor activity of mice was measured using the open field test (OFT) under non-stressed conditions. Briefly, mice were placed individually in an opaque open-field box $(40 \times 40 \times 40 \mathrm{~cm})$ and were allowed to move freely for $15 \mathrm{~min}$. Individual total distance and duration of movement during the first $5 \mathrm{~min}$ were recorded using a video tracking system (EthoVision; Noldus, Wageningen, Netherlands) and were used to assign animals to the test groups, so that the mean distance and duration of movement were similar in all groups. This procedure was used to reduce the initial variability among groups. The mice were again placed in the open field box at $60 \mathrm{~min}$ after the final drug treatment and the total distance and duration of movement were measured during the 5 min OFT. This behavioral experiment was carried out between $13 \mathrm{~h} 00$ and $16 \mathrm{~h} 00$.

Measurement of Serum Corticosterone Blood samples were collected from the iliofemoral artery under light anesthesia immediately before brain fixation. Samples were centrifuged at $4{ }^{\circ} \mathrm{C}$ and sera were stored at $-80^{\circ} \mathrm{C}$ until assayed for corticosterone levels.

Serum corticosterone was measured using the nonradioactive immunoassay AssayMax Corticosterone ELISA Kit (Assaypro, St. Charles, MO, U.S.A.), according to the manufacturer's instructions. The sensitivity of the measurement was $26.99 \mathrm{pg} / \mathrm{ml}$. The intra- and interassay coefficients of variation were 8 and $13 \%$, respectively.

Brain Fixation and Tissue Storage Mice were anesthetized with ether and perfused transcardially with cold phosphate-buffered saline (PBS) and subsequently with cold $4 \%$ paraformaldehyde solution. Brains were collected and postfixed in $4 \%$ paraformaldehyde solution at $4{ }^{\circ} \mathrm{C}$ overnight. Serial coronal sections $(50 \mu \mathrm{m}$ in thickness $)$ were obtained throughout the hippocampus (bregma -1.2 to $-2.5 \mathrm{~mm}$ ) using a vibratome (Technical Products International Inc., St. Louis, MO, U.S.A.) and were stored in $\mathrm{PBS} / \mathrm{NaN}_{3}$ at $4{ }^{\circ} \mathrm{C}$ until needed for subsequent experiments.

Immunohistochemistry All stainings were conducted in 24-well plates for free-floating immunohistochemistry.

For BrdU immunohistochemistry, free-floating sections were incubated in $50 \%$ formamide $/ 2 \times$ saline sodium citrate (SSC) for $2 \mathrm{~h}$ at $65^{\circ} \mathrm{C}$, followed by a rinse in $2 \times \mathrm{SSC}$. Sections were then incubated in $2 \mathrm{~N} \mathrm{HCl}$ for $30 \mathrm{~min}$ at $37^{\circ} \mathrm{C}$ (to denature double-stranded DNA) and rinsed in $0.1 \mathrm{~m}$ borate buffer ( $\mathrm{pH} 8.5$ ). After blocking for $2 \mathrm{~h}$ with $1 \%$ bovine serum albumin (BSA) in PBS containing 0.3\% Triton X-100 (PBS$\mathrm{T})$, sections were incubated overnight at $4{ }^{\circ} \mathrm{C}$ with mouse anti-BrdU monoclonal antibody $(1: 1000$; Chemicon, Temecula, CA, U.S.A.). After rinsing in PBS-T, sections were incubated for $2 \mathrm{~h}$ at room temperature (RT) with biotinylated horse anti-mouse IgG (1:200; Vector Laboratories, Burlingame, CA, U.S.A.), which was followed by incubation with the ABC kit (Vector Laboratories) for $2 \mathrm{~h}$ at RT. BrdUpositive cells were visualized by incubating sections with Vector DAB (Vector Laboratories). Sections were mounted on silane-coated slides and dried, and were then counterstained with $0.05 \%$ toluidine blue (Sigma), dehydrated, and coverslipped using Permount (Fisher Scientific International Inc., Fair Lawn, NJ, U.S.A.).

For OX-A or NPY immunohistochemistry, free-floating sections were incubated in $3 \% \mathrm{H}_{2} \mathrm{O}_{2} / 80 \%$ methanol for $40 \mathrm{~min}$ at RT. After washing in PBS, sections were blocked for $2 \mathrm{~h}$ with $1 \% \mathrm{BSA}$ in PBS-T and incubated for $24 \mathrm{~h}$ at $4{ }^{\circ} \mathrm{C}$ with rabbit anti-OX-A polyclonal antibody (1:1500, Peninsula Laboratories, Inc., San Carlos, CA, U.S.A.) or rabbit anti-NPY polyclonal antibody (1:4000; GeneTex, San Antonio, TX, U.S.A.). Sections were then rinsed in PBS-T, incubated for $2 \mathrm{~h}$ at RT with biotinylated goat anti-rabbit IgG ( $1: 200$; Vector Laboratories), and incubated for $2 \mathrm{~h}$ at RT with the ABC kit. OX-A and NPY-positive cells were visualized by incubating sections with Vector DAB solution. Sections were then mounted and coverslipped as described above.

All counts of BrdU-, OX-A-, or NPY-positive cells were performed on every third section throughout the hippocampus or hypothalamus (bregma -1.2 to $-2.5 \mathrm{~mm}$ ) at $400 \times$ and $1000 \times$ magnification using a light microscope (Olympus BX-41, Tokyo, Japan), to avoid counting the same cells more than once. The number of BrdU-labeled cells in the dentate gyrus of the hippocampus, of OX-A-positive cells in the LHA of the hypothalamus, or of NPY-positive cells in the hilus of the dentate gyrus of each mouse was defined as the total cell counts in six sections. All immunohistochemical procedures included negative controls that lacked primary antibodies. No staining was detected in the controls.

Statistical Analyses Results are presented as means \pm S.E. Statistical analyses were performed using one-way analysis of variance (ANOVA), followed by Tukey's test using Prism (GraphPad Software, San Diego, CA, U.S.A.). Differences were considered significant at $p<0.05$.

\section{RESULTS}

Effect of Kososan on the Stress-Induced Increase in the Duration of Immobility during the FST and on the Spontaneous Locomotor Activity during the OFT The duration of immobility of the stressed mice during the FST was significantly increased when compared with that of nonstressed mice $(p<0.001$, Fig. 1). Oral administration of kososan for $28 \mathrm{~d}$ significantly reduced the stress-induced increase in the duration of immobility, as did the oral administration of milnacipran $(p<0.001)$. Under non-stressed conditions, the oral administration of kososan or milnacipran for $28 \mathrm{~d}$ did not affect the total distance and duration of movement during the OFT (Table 2).

Effect of Kososan on the Number of OX-A-Positive Cells in the Hypothalamus of the Stress-Induced Depression-Like Model Mice OX-A-positive cells were found in the LHA (Fig. 2A). The number of OX-A-positive cells in the stress-induced depression-like model mice was significantly decreased when compared with that of OX-A-positive cells in non-stressed mice $(p<0.01$, Fig. $2 \mathrm{~B})$. Oral administration of kososan or milnacipran for $28 \mathrm{~d}$ significantly reversed the stress-induced decrease in the number of OX-Apositive cells ( $p<0.001$ or $p<0.01$, respectively).

Effect of Kososan on Hippocampal Cell Proliferation 

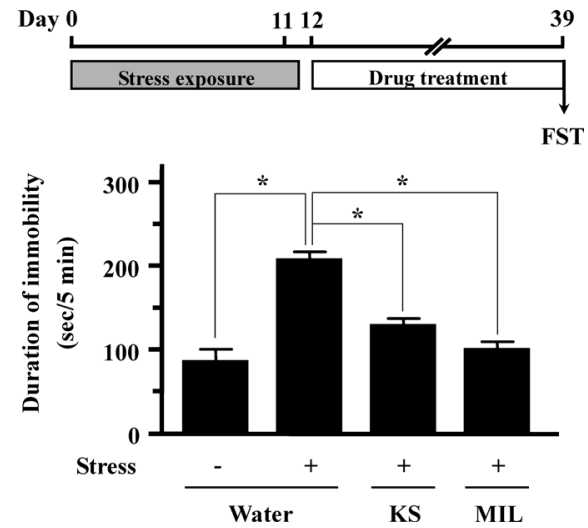

Fig. 1. Effect of Kososan on the Stress-Induced Increase in the Duration of Immobility during the FST

Kososan $(1.0 \mathrm{~g} / \mathrm{kg})$ or milnacipran $(60 \mathrm{mg} / \mathrm{kg})$ were administered orally to stress-induced depression-like model mice once daily for $28 \mathrm{~d}$. The duration of immobility was measured during a 5 min FST at 60 min after the final drug treatment. Each column represents the mean \pm S.E. $(n=12-22)$. $* p<0.001$ with Tukey's test. FST, forced swimming test; KS, kososan; MIL, milnacipran
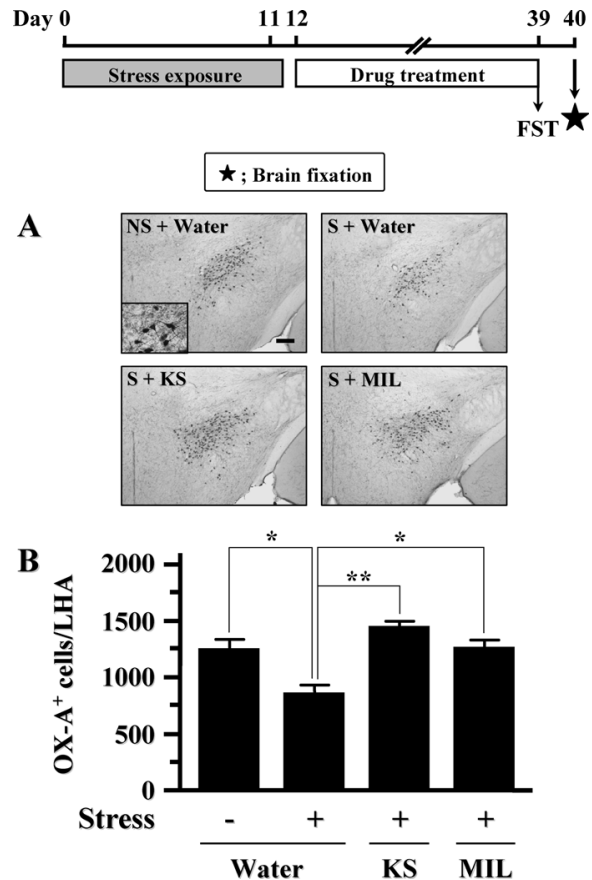

Fig. 2. Effect of Kososan on OX-A Expression in the LHA of the StressInduced Depression-Like Model Mice

Kososan $(1.0 \mathrm{~g} / \mathrm{kg})$ or milnacipran $(60 \mathrm{mg} / \mathrm{kg})$ were administered orally to stress-induced depression-like model mice once daily for $28 \mathrm{~d}$. OX-A immunohistochemistry was performed on brain slices prepared from animals that were sacrificed $1 \mathrm{~d}$ after the FST. (A) Photomicrographs of the LHA in the hypothalamus showing representative OX-A-positive cells. The small panel shows a high magnification $(400 \times)$ of OX-A positive cells. Scale bar $=100 \mu \mathrm{m}$. (B) The number of OX-A-positive cells in the LHA was counted. Each column represents the mean \pm S.E. $(n=19-20) . * p<0.01$ and $* * p<0.001$ with Tukey's test. FST, forced swimming test; OX-A, orexin-A; NS, nonstress; S; stress; KS, kososan; MIL, milnacipran; LHA, lateral hypothalamic area

and Serum Corticosterone Levels in the Stress-Induced Depression-Like Model Mice The number of BrdU-positive cells in the stress-induced depression-like model mice was significantly decreased when compared with that of BrdU-positive cells in non-stressed mice $(p<0.05$, Figs. 3A and B). Oral administration of kososan or milnacipran for $28 \mathrm{~d}$ significantly attenuated the stress-induced decrease in the number of BrdU-positive cells $(p<0.05)$.
Table 2. Effect of Kososan on the Spontaneous Locomotor Activities in the OFT

\begin{tabular}{lcc}
\hline \hline & $\begin{array}{c}\text { Total distance } \\
(\mathrm{cm} / 5 \mathrm{~min})\end{array}$ & $\begin{array}{c}\text { Total duration } \\
(\mathrm{s} / 5 \mathrm{~min})\end{array}$ \\
\hline Water & $1464 \pm 104.5$ & $172.1 \pm 10.9$ \\
KS $(1.0 \mathrm{~g} / \mathrm{kg})$ & $1465 \pm 112.8$ & $174.6 \pm 12.8$ \\
MIL $(60 \mathrm{mg} / \mathrm{kg})$ & $1320 \pm 154.0$ & $162.1 \pm 16.7$ \\
\hline
\end{tabular}

Kososan $(1.0 \mathrm{~g} / \mathrm{kg})$ or milnacipran $(60 \mathrm{mg} / \mathrm{kg})$ were administered orally to non stressed mice once daily for $28 \mathrm{~d}$. Total distance and duration of movement were measured during a $5 \mathrm{~min}$ OFT at $60 \mathrm{~min}$ after the final administration of kososan or milnacipran. Each value represents the mean \pm S.E. $(n=11)$. OFT, open field test; KS, kososan; MIL, milnacipran.
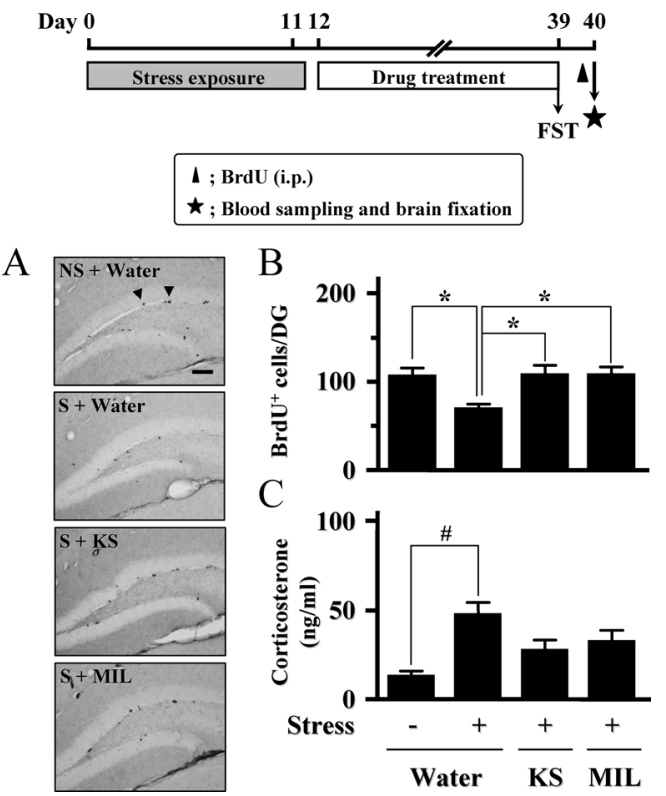

Fig. 3. Effect of Kososan on Hippocampal Cell Proliferation and Serum Corticosterone Levels in the Stress-Induced Depression-Like Model Mice

Kososan $(1.0 \mathrm{~g} / \mathrm{kg})$ or milnacipran $(60 \mathrm{mg} / \mathrm{kg})$ were administered orally to stress-induced depression-like model mice once daily for $28 \mathrm{~d}$. BrdU $(200 \mathrm{mg} / \mathrm{kg})$ was injected once at $4 \mathrm{~h}$ before brain fixation and BrdU immunohistochemistry was performed on brain slices prepared from animals that were sacrificed $1 \mathrm{~d}$ after the FST. (A) Photomicrographs of dentate gyrus showing representative BrdU-positive cells, which are indicated by arrowheads. Scale bar $=100 \mu \mathrm{m}$. (B) The number of BrdU-positive cells in the dentate gyrus was counted. (C) Blood samples were collected immediately before brain fixation. Serum corticosterone levels were measured using a corticosterone ELISA kit. Each column represents the mean \pm S.E. (B, $n=10-11 ; \mathrm{C}, n=8) . * p<0.001$ and ${ }^{\#} p<0.01$ with Tukey's test. FST, forced swimming test; BrdU, bromodeoxyuridine; DG, dentate gyrus; NS, non-stress; S, stress; KS, kososan; MIL, milnacipran.

The serum corticosterone levels were significantly increased in the stress-induced depression-like model mice when compared with those of the non-stressed mice $(p<0.01$, Fig. 3C). Oral administration of kososan or milnacipran for $28 \mathrm{~d}$ showed a tendency to decrease the stressinduced increase in corticosterone levels.

Effect of SB-334867 on the Kososan-Induced Reduction of Immobility in the Stress-Induced Depression-Like Model Mice SB-334867 potently blocked the kososan-induced decrease in immobility during the FST $(p<0.01$, Fig. 4). In contrast, SB-334867 failed to block the milnacipran-induced decrease in immobility. The duration of immobility was not affected by the treatment with SB-334867 in the stress-induced depression-like model mice.

Effect of SB-334867 on the Kososan-Induced Increase in Cell Proliferation in the Dentate Gyrus of the Stress- 

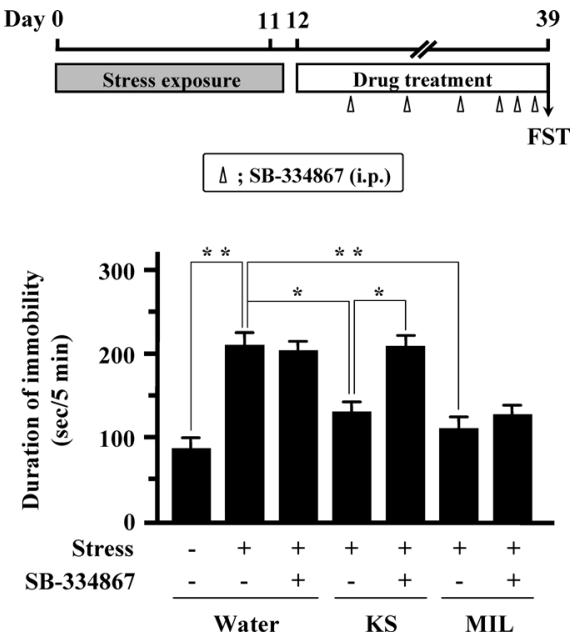

Fig. 4. Effect of SB-334867 on the Kososan-Induced Reduction of Immobility in the Stress-Induced Depression-Like Model Mice

Kososan $(1.0 \mathrm{~g} / \mathrm{kg})$ or milnacipran $(60 \mathrm{mg} / \mathrm{kg})$ were administered orally to stress-induced depression-like model mice once daily for $28 \mathrm{~d}$. SB-334867 $(15 \mathrm{mg} / \mathrm{kg})$ was in jected once daily on Days $18,25,32,35,37$, and 39 . The duration of immobility was measured during a $5 \mathrm{~min}$ FST at $90 \mathrm{~min}$ after the final injection of SB-334867. Each column represents the mean \pm S.E. $(n=11-12) . * p<0.01$ and $* * p<0.001$ with Tukey's test. FST, forced swimming test; KS, kososan; MIL, milnacipran.
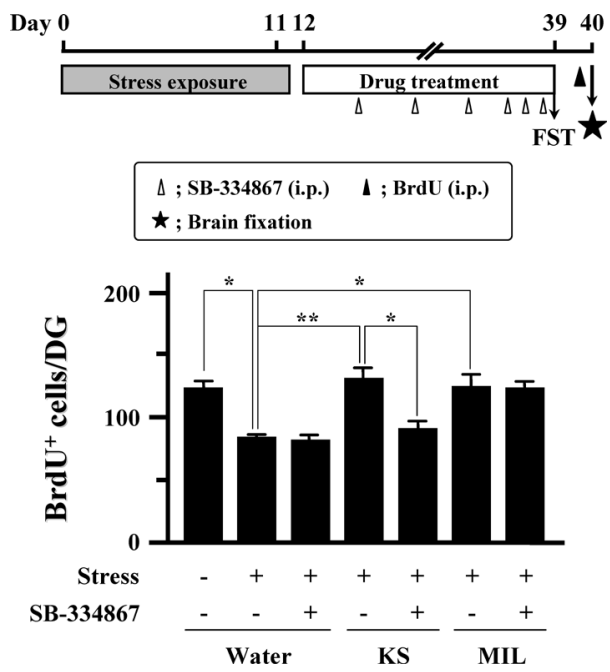

Fig. 5. Effect of SB-334867 on the Kososan-Induced Increase in Hippocampal Cell Proliferation in the Stress-Induced Depression-Like Model Mice

Kososan $(1.0 \mathrm{~g} / \mathrm{kg})$ or milnacipran $(60 \mathrm{mg} / \mathrm{kg})$ were administered orally to stress-induced depression-like model mice once daily for $28 \mathrm{~d}$. SB-334867 $(15 \mathrm{mg} / \mathrm{kg})$ was injected once daily on Days $18,25,32,35,37$, and 39 . BrdU $(200 \mathrm{mg} / \mathrm{kg})$ was injected once at $4 \mathrm{~h}$ before brain fixation. BrdU immunohistochemistry was performed on brain slices prepared from animals that were sacrificed $1 \mathrm{~d}$ after the FST. The number of BrdU-positive cells in the dentate gyrus was counted. Each column represents the mean \pm S.E. $(n=5-6) . * p<0.05$ and $* * p<0.01$ with Tukey's test. FST, forced swimming test; BrdU, bromodeoxyuridine; DG, dentate gyrus; KS, kososan; MIL, milnacipran

Induced Depression-Like Model Mice SB-334867 significantly blocked the kososan-induced increase in the number of BrdU-positive cells in the stress-induced depression-like model mice $(p<0.01$, Fig. 5). In contrast, SB-334867 failed to block the milnacipran-induced increase in the number of BrdU-positive cells. The number of BrdU-positive cells was not affected by the treatment with SB-334867 in the stressinduced depression-like model mice.

Effect of Kososan on the Number of NPY-Positive Cells in the Dentate Gyrus of the Hippocampus of the Stress-
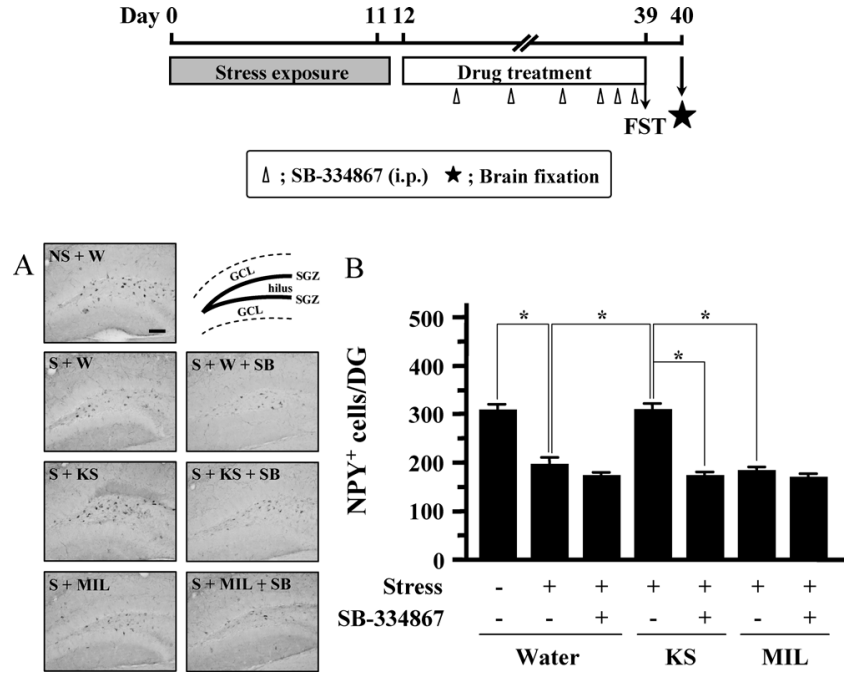

Fig. 6. Effect of Kososan on Hippocampal NPY Expression in the StressInduced Depression-Like Model Mice

Kososan $(1.0 \mathrm{~g} / \mathrm{kg})$ or milnacipran $(60 \mathrm{mg} / \mathrm{kg})$ were administered orally to stress-induced depression-like model mice once daily for $28 \mathrm{~d}$. SB-334867 $(15 \mathrm{mg} / \mathrm{kg})$ was in jected once daily on Days 18, 25, 32, 35, 37, and 39. NPY immunohistochemistry was performed on brain slices prepared from animals that were sacrificed $1 \mathrm{~d}$ after the FST. (A) Photomicrographs of the dentate gyrus showing representative NPY-positive cells. The illustration represents several regions of the granule cell layer (GCL), subgranular zone (SGZ), and hilus in the dentate gyrus. Scale bar $=100 \mu \mathrm{m}$. (B) The number of NPY-positive cells in the dentate gyrus was counted. Each column represents the mean \pm S.E $(n=11-12) . * p<0.001$ with Tukey's test. W, water; SB, SB-334867. FST, forced swimming test; DG, dentate gyrus; NS, non-stress; S, stress; KS, kososan; MIL, milnacipran; NPY, neuropeptide Y.

Induced Depression-Like Model Mice Most NPY-positive cells in the dentate gyrus were localized in the hilus and subgranular zone (SGZ) (Fig. 6A). The number of NPY-positive cells in the dentate gyrus in the stress-induced depression-like model mice was significantly decreased when compared with that of NPY-positive cells in non-stressed mice $(p<0.001$, Fig. 6B). Oral administration of kososan for $28 \mathrm{~d}$ significantly reversed the stress-induced decrease in the number of NPY-positive cells in the dentate gyrus $(p<0.001)$ and SB-334867 significantly blocked the kososan-induced increase in the number of NPY-positive cells $(p<0.001)$. However, oral administration of milnacipran for $28 \mathrm{~d}$ failed to reverse the stress-induced decrease in the number of NPY-positive cells.

\section{DISCUSSION}

Our results demonstrate that oral administration of kososan for $28 \mathrm{~d}$ exerted a therapeutic antidepressive-like effect in the stress-induced depression-like model mice and that kososan and milnacipran alleviated the stress-induced decrease in the cell proliferation in the dentate gyrus of the hippocampus. More interestingly, the antidepressive-like effect of kososan, in contrast to what was observed for milnacipran, may be closely associated with the regulation of the orexinergic system and with, in part, the mediation of NPYergic system.

Regarding the experimental design of the present study, the reexposure of mice to a stressful event, such as forced swimming on $28 \mathrm{~d}$ after exposure to the stresses, led to a depressive-like behavior (e.g., immobilized condition in the FST), which suggests that spontaneous recovery was not observed after a recovery period of $28 \mathrm{~d}$. This result implies that 
stress vulnerability may persist beyond the period of stress exposure. In the present study, therefore, we attempted to examine the therapeutic effects of drugs during the period of recovery from depressive states.

Oral administration of kososan for $28 \mathrm{~d}$ reduced the duration of immobility during the FST without affecting the spontaneous locomotor activity, which suggests that chronic treatment with kososan exerts an antidepressive-like effect. Our previous study showed that subchronic treatment with kososan for $9 \mathrm{~d}$ produced an antidepressive-like effect in the stress-induced depression-like model mice when drug treatment was performed in parallel with exposure to the stresses. ${ }^{2}$ This finding may mean that kososan has a preventive antidepressive-like effect. In addition, the results of the present study demonstrated that kososan possessed a therapeutic antidepressive-like effect, as the 28-d drug treatment was performed after exposure to the stresses. Our results also appear to be supported by the finding that the onset of the therapeutic clinical effects of certain antidepressants (e.g., tricyclic antidepressants and selective serotonin reuptake inhibitors) in patients with depression is usually observed at least 3 to 4 weeks after the initiation of treatment. ${ }^{32)}$ Therefore, these findings may constitute evidence for the efficacy of kososan against stress and depression.

The number of OX-A-positive cells in the LHA was decreased in the stress-induced depression-like model mice. This result is supported by the observation that the decreased function of the orexinergic system is involved in the pathology of depression. ${ }^{15,16)}$ Oral administration of kososan for $28 \mathrm{~d}$ also significantly reversed the stress-induced decrease in the number of OX-A-positive cells in the LHA. Our preliminary study demonstrated that the number of OX-A-positive cells in the LHA was decreased just after exposure to stresses when compared with non-stressed mice (data not shown). These findings suggest that treatment with kososan for $28 \mathrm{~d}$ may bring the levels of OX-A in the LHA to the normal condition.

BrdU labeling is widely used in neurogenesis-related studies, as BrdU, which is an analogue of thymidine, is rapidly incorporated into dividing and proliferating cells. Kososan treatment significantly attenuated the stress-induced decrease in the number of BrdU-positive cells in the dentate gyrus $4 \mathrm{~h}$ after BrdU injection, which suggests that oral administration of kososan for $28 \mathrm{~d}$ increases hippocampal cell proliferation. We have previously demonstrated that the proliferation of cells in the hippocampus just after exposure to stresses was not affected when compared with what was observed for non-stressed mice; however, cell proliferation during the recovery period after exposure to stresses was decreased in a time-dependent manner (data not shown). Therefore, the present result suggests that oral administration of kososan for $28 \mathrm{~d}$ may suppress the stress-induced decrease in cell proliferation during the recovery period. Interestingly, the effect of kososan on hippocampal cell proliferation was apparently similar to that of milnacipran; the effect of milnacipran on cell proliferation has not been previously reported. Our results may confirm further the antidepressive-like effects of cell proliferation-inducing drugs. ${ }^{33-35)}$

The kososan-induced antidepressive-like effect and the increase in cell proliferation were blocked by treatment with SB-334867, which is an OXR1 antagonist. The inhibitory ef- fect of SB-334867 on kososan-induced cell proliferation appears to be, at least in part, consistent with our previous study, which showed that OX-A induced hippocampal cell proliferation. ${ }^{17)}$ Therefore, these results suggest that the kososan-induced alteration of the orexinergic system in the stress-induced depression-like model mice may regulate the antidepressive-like effect of the drug and the promotion of cell proliferation via OXR1. The elevated levels of serum corticosterone found here and the corresponding decrease in cell proliferation are also consistent with well-established findings of suppression of hippocampal cell proliferation by corticosterone. ${ }^{10,36)}$ However, the stress-induced increase in the levels of corticosterone was not significantly attenuated in mice treated with kososan. A possible cause for the fact that oral administration of kososan significantly inhibited the stress-induced decrease in cell proliferation is not able to explain only by the control of serum corticosterone levels. The kososan-induced upregulation of the OX-A system is thought to compensate for the lack of normalization in the cell proliferation that is accompanied by a decrease in corticosterone levels. In addition, this interaction may lead to the kososaninduced alleviation of the decrease in cell proliferation. It is noteworthy that treatment with SB-334867 failed to block the milnacipran-induced antidepressive-like effect and the increase in cell proliferation, although oral administration of both milnacipran and kososan reversed the stress-induced decrease in OX-A levels. These results suggest that the milnacipran-induced antidepressive-like effect and the increase in hippocampal cell proliferation were, at least in part, independent from OXR1 signaling.

We have previously demonstrated that the antidepressivelike effect of OX-A may correlate with the regulation of the NPY system in the dentate gyrus via OXR1 signaling. ${ }^{17)}$ This finding is supported by the report that NPY neurons are directly activated by OX-A via OXR $\left.1 .{ }^{37}\right)$ Thus, we investigated whether kososan treatment modifies NPYergic transmission in the dentate gyrus. In the present study, the number of NPY-positive cells in the dentate gyrus was decreased in the stress-induced depression-like model mice. This result is supported by recent studies that showed that animal models of depression (e.g., chronic unpredicted stress, learned helplessness, and maternal separation) display a significant decrease in the hippocampal levels of NPY. ${ }^{27,38,39)}$ Moreover, a genetic animal model of depression, such as the Flinders sensitive line rat, also exhibits a significant decrease in the hippocampal levels of NPY. ${ }^{40)}$ Oral administration of kososan for $28 \mathrm{~d}$ also ameliorated the stress-induced decrease in the number of NPY-positive cells. Moreover, the kososan-induced increase in the number of NPY-positive cells was completely blocked by treatment with SB-334867. This result indicates that kososan induced the expression of NPY in the dentate gyrus through the orexinergic system. Our previous in vitro study demonstrated that OX-A does not have a proliferative effect on rat fetal brain-derived neural progenitor cells that do not express the OXR1 protein. ${ }^{17}$ ) However, several studies have demonstrated that the Y1 receptor, which is a subtype of NPY receptor, is expressed in neural progenitor cells, ${ }^{25}$ ) where NPY functions directly as a neuroproliferative factor. ${ }^{41-43)}$ Our results, together with these findings, raise the hypothesis that the kososan-induced upregulation of OXA expression may lead to an increase in cell proliferation via 
the elevation of the levels of NPY expression in the dentate gyrus. However, it remains unclear whether the kososaninduced increase in NPY expression is directly or indirectly related to the antidepressive-like effect and the regulation of cell proliferation. A recent study demonstrated that infusion of NPY into the hippocampus produces an antidepressivelike activity via the Y1 receptor. ${ }^{44)}$ Hence, further studies on the effects of a Y1 receptor antagonist on the kososaninduced antidepressive-like effect and cell proliferation may help to further clarify the roles of NPYergic transmission in the mediation of the kososan-induced effects. In contrast, the number of NPY-positive cells was not affected by the milnacipran treatment. This result suggests that milnacipran does not regulate the expression of NPY in the dentate gyrus. More recently, it was demonstrated that fluoxetine, which is a selective serotonin reuptake inhibitor, exhibits antidepressive-like and cell proliferation-inducing effects, without the intervention of NPYergic transmission. ${ }^{45}$ This observation suggests that it is necessary to examine whether the antidepressive-like effect of milnacipran is caused by cell proliferation-inducing effects that are independent of the regulation of the NPYergic transmission.

Collectively, this study provides additional evidence that long-term administration of kososan exerts a therapeutic antidepressive-like effect in the stress-induced depression-like model mice and suppresses the stress-induced reduction of cell proliferation via the regulation of the orexinergic system and/or NPYergic transmission. These findings may contribute to the exploration of the potential differences in the mechanisms underlying the antidepressive-like effects of kososan and of antidepressants.

Acknowledgments We would like to thank Ms. C. Kato for technical assistance.

\section{REFERENCES}

1) Hanawa T., Journal of Kampo Medicine, 42, 418-426 (1995).

2) Ito N., Nagai T., Yabe T., Nunome S., Hanawa T., Yamada H., Phytomedicine, 13, 658-667 (2006).

3) Nagai T., Narikawa T., Ito N., Takeda T., Hanawa T., Yamada H., J. Trad. Med., 25, 74-80 (2008).

4) Holsboer F., Neuropsychopharmacology, 23, 477-501 (2000).

5) Kendler K. S., Karkowski L. M., Prescott C. A., Am. J. Psychiatry, 156, 837-841 (1999).

6) Holsboer F., Barden N., Endocr. Rev., 17, 187-205 (1996).

7) Joels M., Karst H., Alfarez D., Heine V. M., Qin Y., van Riel E., Verkuyl M., Lucassen P. J., Krugers H. J., Stress, 7, $221-231$ (2004).

8) Gold P. W., Chrousos G. P., Mol. Psychiatry, 7, 254-275 (2002).

9) Duman R. S., Malberg J., Thome J., Biol. Psychiatry, 46, 1181-1191 (1999).

10) Tanapat P., Galea L. A., Gould E., Int. J. Dev. Neurosci., 16, 235-239 (1998).

11) Malberg J. E., Eisch A. J., Nestler E. J., Duman R. S., J. Neurosci., 20, 9104-9110 (2000)

12) Santarelli L., Saxe M., Gross C., Surget A., Battaglia F., Dulawa S., Weisstaub N., Lee J., Duman R., Arancio O., Belzung C., Hen R., Science, 301, 805-809 (2003).

13) Peyron C., Tighe D. K., van den Pol A. N., de Lecea L., Heller H. C., Sutcliffe J. G., Kilduff T. S., J. Neurosci., 18, 9996-10015 (1998).

14) Nambu T., Sakurai T., Mizukami K., Hosoya Y., Yanagisawa M., Goto
K., Brain Res., 827, 243-260 (1999).

15) Allard J. S., Tizabi Y., Shaffery J. P., Trouth C. O., Manaye K., Neuropeptides, 38, 311-315 (2004)

16) Brundin L., Bjorkqvist M., Petersen A., Traskman-Bendz L., Eur. Neuropsychopharmacol., 17, 573-579 (2007).

17) Ito N., Yabe T., Gamo Y., Nagai T., Oikawa T., Yamada H., Hanawa T., Neuroscience, 157, 720-732 (2008).

18) Hervieu G. J., Cluderay J. E., Harrison D. C., Roberts J. C., Leslie R. A., Neuroscience, 103, 777-797 (2001).

19) Clark J. T., Kalra P. S., Kalra S. P., Endocrinology, 117, 2435-2442 (1985).

20) Kharlamov E. A., Kharlamov A., Kelly K. M., Brain Res., 1127, 151162 (2007).

21) Widerlov E., Lindstrom L. H., Wahlestedt C., Ekman R., J. Psychiatr Res., 22, 69-79 (1988).

22) Gjerris A., Widerlov E., Werdelin L., Ekman R., J. Psychiatry Neurosci., 17, 23-27 (1992).

23) Heilig M., Zachrisson O., Thorsell A., Ehnvall A., Mottagui-Tabar S., Sjogren M., Asberg M., Ekman R., Wahlestedt C., Agren H., J. Psychiatr. Res., 38, 113-121 (2004).

24) Redrobe J. P., Dumont Y., Fournier A., Quirion R., Neuropsychopharmacology, 26, 615-624 (2002).

25) Howell O. W., Scharfman H. E., Herzog H., Sundstrom L. E., BeckSickinger A., Gray W. P., J. Neurochem., 86, 646-659 (2003).

26) Zhang Y. Z., Li Y. F., Yu N. J., Yuan L., Zhao Y. M., Xiao W. B., Luo Z. P., Chin. Med. J. (Engl), 120, 1792-1796 (2007).

27) An L., Zhang Y. Z., Yu N. J., Liu X. M., Zhao N., Yuan L., Chen H. X., Li Y. F., Prog. Neuropsychopharmacol. Biol. Psychiatry, 32, 1484-1490 (2008)

28) Porsolt R. D., Bertin A., Jalfre M., Arch. Int. Pharmacodyn. Ther, 229 327-336 (1977).

29) Detke M. J., Johnson J., Lucki I., Exp. Clin. Psychopharmacol., 5, 107-112 (1997)

30) Solberg L. C., Horton T. H., Turek F. W., Am. J. Physiol., 276, R152161 (1999).

31) Willner P., Towell A., Sampson D., Sophokleous S., Muscat R., Psychopharmacology (Berlin), 93, 358-364 (1987).

32) Wong M. L., Licinio J., Nat. Rev. Neurosci., 2, 343-351 (2001).

33) Lee H. J., Kim J. W., Yim S. V., Kim M. J., Kim S. A., Kim Y. J., Kim C. J., Chung J. H., Mol. Psychiatry, 6, 610, 725-618 (2001).

34) Alonso R., Griebel G., Pavone G., Stemmelin J., Le Fur G., Soubrie P., Mol. Psychiatry, 9, 278-286, 224 (2004).

35) Li Y. F., Zhang Y. Z., Liu Y. Q., Wang H. L., Yuan L., Luo Z. P., Acta Pharmacol. Sin., 25, 1408-1412 (2004).

36) Cameron H. A., Gould E., Neuroscience, 61, 203-209 (1994).

37) Muroya S., Funahashi H., Yamanaka A., Kohno D., Uramura K., Nambu T., Shibahara M., Kuramochi M., Takigawa M., Yanagisawa M., Sakurai T., Shioda S., Yada T., Eur. J. Neurosci., 19, 1524-1534 (2004).

38) Ishida H., Shirayama Y., Iwata M., Kawahara R., Brain Res., 1046, $239-243$ (2005)

39) Jimenez-Vasquez P. A., Mathe A. A., Thomas J. D., Riley E. P., Ehler C. L., Brain Res. Dev. Brain Res., 131, 149-152 (2001).

40) Jimenez-Vasquez P. A., Diaz-Cabiale Z., Caberlotto L., Bellido I., Overstreet D., Fuxe K., Mathe A. A., Eur. Neuropsychopharmacol., 17, 298-308 (2007)

41) Hansel D. E., Eipper B. A., Ronnett G. V., Nature (London), 410, $940-944$ (2001).

42) Howell O. W., Doyle K., Goodman J. H., Scharfman H. E., Herzog H., Pringle A., Beck-Sickinger A. G., Gray W. P., J. Neurochem., 93, $560-570$ (2005).

43) Howell O. W., Silva S., Scharfman H. E., Sosunov A. A., Zaben M., Shatya A., McKhann G., 2nd, Herzog H., Laskowski A., Gray W. P., Neurobiol. Dis., 26, 174-188 (2007).

44) Ishida H., Shirayama Y., Iwata M., Katayama S., Yamamoto A., Kawahara R., Nakagome K., Hippocampus, 17, 271-280 (2007).

45) Karlsson R. M., Choe J. S., Cameron H. A., Thorsell A., Crawley J. N., Holmes A., Heilig M., Psychopharmacology (Berlin), 195, 547-557 (2008). 